\title{
Assessment of Toxicity and Drug-Drug Interaction of Combination Therapy Prescribed by Physicians/ Clinicians for Treatment of Diabetes using Experimental Animals
}

\author{
Hemlata Dewangan', Raj Kumar Tiwari², Vikas Sharma', Shiv Shankar Shukla', \\ Ravindra Kumar Pandey ${ }^{1, *}$
}

${ }^{1}$ Department of Pharmacology, Columbia Institute of Pharmacy, Raipur, Chhattisgarh, INDIA.

${ }^{2}$ Columbia Institute of Pharmacy, Raipur, Chhattisgarh, INDIA.

\begin{abstract}
Purpose: The management of diabetes associated with hyperlipidemia is a challenge not only to the physician but also to medical fraternity. Hence most of the physicians prefer to prescribe antihyperglycemic drug along with some lipid lowering agent. The present study is aimed to find out toxicity and drug interaction of combination therapy of hypoglycemic agents (Glimepride, Metformin) withhypolipidemic drugs (Atovastatin + Fenofibrate). Materials and Methods: Young healthy adult Albino Wister rats (150-180 g) were used in the study. The experimental animals we reassessed for acute toxicity study and ant diabetic activity. The drug-drug interaction was also evaluated by changes in physiological, hematological parameters and histopathology of different tissues. Results: The result of acute toxicity revealed that there was a change in body weight in the animal treated with hypoglycemic agents (Glimepride, Metformin) withhypolipidemic drugs (Atovastatin + Fenofibrate). Water, food consumption has not been varied with greater extent in all groups of animals. Mild changes were marked inhemoglobin, neutrophils, platelet count. Conclusion: The combination of drugs showed mild change in the effect of Glimepride + Metformin alone and in combination with Atorvastatin + Fenofibrate. Other factors showed no significant effect which proves that there is no drug interaction.
\end{abstract}

Key words: Drug interaction, Diabetes, Toxicity, Combination therapy, Metformin, Atorvastatin.

\section{INTRODUCTION}

An alteration in the effectiveness or toxicity of one drug due to another simultaneously administered drug is known as drug-drug interaction. A drug interaction may result in adverse effect where in there is a decrease in the effectiveness or an increase in the toxicity of one drug in the presence of another drug. Biological interference with laboratory test may mislead the diagnosis. Beneficial effects, where in there is an increase in the effectiveness or a decrease in the toxicity of one drug in the presence of another. Pharmacokinetic interaction is those in which absorption, distribution, metabolism, excretion of a drug is altered. ${ }^{1-4}$
The drug interaction studies assume much importance, especially for drugs that have a narrow margin of safety and where the drugs are used for a prolonged period of time. ${ }^{5}$ Drug-drug interaction can be defined as the modification of the effects of one drug (i.e., the object drug) by the prior or concomitant administration of another drug. ${ }^{6}$ Diabetes mellitus often referred to simply as diabetes, is a syndrome of disordered metabolism, usually due to a combination of hereditary and environmental causes, resulting in abnormally high blood sugar levels (hyperglycemia). Blood glucose levels are controlled by a complex interaction
Submission Date: 01-07-2020; Revision Date: 02-12-2020; Accepted Date: 21-04-2021

DOI: 10.5530/ijper.55.2s.133 Correspondence:

Dr. Ravindra Kumar Pandey Department of Pharmacology, Columbia Institute of Pharmacy, Raipur-493111, Chhattisgarh, INDIA.

Phone no: +91-9826229321 Email id: ravindraiop@gmail. com

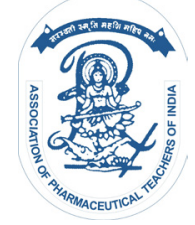

www.ijper.org 
of multiple chemicals and hormones in the body, including the hormone insulin made in the beta cells of the pancreas. Diabetes mellitus refers to the group of diseases that lead to high blood glucose levels due to defects in either insulin secretion or insulin action. ${ }^{7}$ Diabetes mellitus is a chronic major endocrine disorder and growing health problem in most countries and is characterized by hyperglycemia, hyperlipemia, negative nitrogen balance, oxidative stress and sometimes ketonemia. The terms "Diabetes" and "Mellitus" are derived from Greek. "Diabetes" denotes "a passer through; a siphon" whereas the "Mellitus" denotes "sweet". ${ }^{-11}$

Type-1 Diabetes is characterized by autoimmune destruction of insulin producing cells in the pancreas by $\mathrm{CD} 4+$ and $\mathrm{CD} 8+\mathrm{T}$ cells and macrophages infiltrating the islets. ${ }^{12-14}$ In type-2 diabetes, these mechanisms break down, with the consequence that the two main pathological defects in type- 2 diabetes are impaired insulin secretion through a dysfunction of the pancreatic $\beta$ - cell and impaired insulin action through insulin resistance. ${ }^{15,16}$

Insulin plays an important role in the regulation of intermediary lipid metabolism and fluctuations in the degree of diabetic control thus produce variable effects on plasma lipoprotein metabolism. Non-insulin-dependent (Type 2) diabetic patients are obese and obesity may lead to the development of hyperlipidemia. ${ }^{1718}$ The relationship between diabetes and hyperlipidemia is due to the unusually high prevalence of accelerated atherosclerosis in diabetic patients. ${ }^{19-21}$ This is usually caused by the accumulation of very low-density lipoprotein (VLDL) and, rarely, chylomicrons in the plasma. ${ }^{22-25}$

The aim of the present study was to evaluate the possible drug interactions in the prescribed combination therapy in a diabetic patient associated with hyperlipidemia using experimental animals (rats) and make researchers aware of them so as to prevent the occurrence/ reoccurrence of clinical adverse events. Another objective of the study is to provide a basic of drug interaction of widely used diabetes drug therapy, to young physicians and researchers.

\section{MATERIALS AND METHODS}

Drugs and Chemicals: Glimepride $(1 \mathrm{mg})$ and Metformin (500mg) was procured from local chemical suppliers. Atorvastatin (10mg) and Fenofibrate (145mg) combination with were also procured from local chemical suppliers. All other chemicals used were of analytical grade.

\section{Experimental animals}

Young and healthy Albino Wistar Rats weight between 150-180 gm of either sex was procured from Institutional animal ethics committee (IAEC) approved by CPCSEA, Columbia Institute of Pharmacy, Raipur (C.G.) approval No - CIP/IAEC/2017/085 and Regd. No.1321/PO/ReBi/S/10/CPCSEA. The animals were housed in polypropylene cages and allowed to acclimatize to laboratory conditions for at least 7 days. Animals were randomly assigned to various groups. All the animals were maintained under standard husbandry conditions of 12:12 h light: dark cycle at a temperature of $24 \pm 2{ }^{\circ} \mathrm{C}$ and a relative humidity of $30-70 \%$ with free access to water and standard laboratory rat pellet diet. All the animal handling and blood with drawl techniques were carried out in accordance with the Committee for the purpose of control and supervision on experimental animals (CPCSEA) guidelines.

\section{Grouping of Animals}

The animals (Wistar rats) of either sex (male -female animals) randomly divided into 4 groups. Number of animals in each group $=10$ i.e. $($ Male $=5$, Female $=5$ ).

\begin{tabular}{|c|c|}
\hline Group I & Control group (Received Vehicle) \\
\hline Group II & $\begin{array}{c}\text { Control' (Administered with Glimepride + } \\
\text { Metformin) }\end{array}$ \\
\hline Group III & $\begin{array}{c}\text { Test group (Administered with Atovastatin + } \\
\text { Fenofibrate) }\end{array}$ \\
\hline Group IV & $\begin{array}{c}\text { Test group (Combination of Glimepride + } \\
\text { Metformin -Atovastatin + Fenofibrate.) }\end{array}$ \\
\hline
\end{tabular}

\section{Acute Oral Toxicity Study}

The acute toxicity of marketed formulation was evaluated as per OECD Guideline-407. The four groups were selected and ten animals were present in each group. Animals received dose of Glimepride + Metformin - Atorvastatin + Fenofibrate in Wistar rat orally after a short fasting period using oral gavages. The general behavior of the animal was continuously monitored for $1 / 2 \mathrm{hr}, 1 \mathrm{hr}, 2 \mathrm{hr}$ and $3 \mathrm{hr}$ after dosing, periodically during the first $24 \mathrm{hr}$ (with special attention given during the first $4 \mathrm{hr}$ )and the same treatment was followed for 7 days.

\section{Cage-side observations}

The detailed cage-side observations were observed including changes in mucous membranes, Tremor, Convulsion, salivation, diarrhea, lethargy, sleep and also motor activity and behavior pattern. All rats were 
observed for toxic signs and any pre-terminal deaths daily.

\section{Body weight, food and water intake}

Food and water intake was recorded daily and average weekly consumption was calculated. Individual body weight was recorded once in a week.

\section{Histopathology}

Liver, Kidney and Heart were fixed immediately in 10\% formalin for routine Histopathological examination. The tissues were embedded in paraffin and then sectioned, stained with Haematoxylin and Eosin and were examined under light microscope.

\section{Repeated dose 28 days Oral Dose Toxicity}

A repeated toxicity study of the same drug was conducted on male albino wistar rats for a period of 28 days according to the OECD-407 Guideline. The animals were divided into 4 groups (control, control* Test, Test*), each group containing ten animals. While the drug was orally administered using gavage to test groups, the vehicle was administered to the control group for 28 days. All animals were supplied with standard food and water ad libitum during the testing periods. All rats were observed daily for toxic manifestations and mortality. Body weight, water and food intake were measured on a daily basis.

\section{Hematology}

Blood was collected by Retro orbital plexus from the overnight fasted animals. Investigation of whole blood for following was done: Hemoglobin ( $\mathrm{Hb})$, Platelet count, Neutrophils (N), Lymphocytes (L) and lymphocyte.

\section{Therapeutic effect}

\section{Combination therapy}

The second set of experiment was carried out on animals (wistar rats) to determine therapeutic effectiveness of combination therapy prescribed by register medical practitioner in different clinics. In set of experiment rats either sex are randomly divided into one group containing 6 animals. All the animals were administered Streptozotocin + High fat diet to induce diabetes associate with hyperlipidemia.

\section{Induction of diabetes}

Streptozotocin (STZ) induced hyperglycemia has been described as a useful experimental model to study the activity of hypoglycemic agents. After overnight fasting (deprived of food for $16 \mathrm{hrs}$, had been allowed free access to water), diabetes was induced in rats by intraperitoneal injection of STZ dissolved in $0.1 \mathrm{M}$ sodium citrate buffer $\mathrm{pH} 4.5$ at a dose of $50 \mathrm{mg} / \mathrm{kg}$ body weight. After the injection, free access for food and water was provided. The development of diabetes was confirmed after $48 \mathrm{~h}$ of the streptozotocin injection. The animals having fasting blood glucose levels more than $200 \mathrm{mg} / \mathrm{dL}$ were considered as diabetic rats and used for experimentation.

\section{Study Procedure}

Blood samples were collected from the tail vein at time intervals after drug administration and glucose levels were estimated by using glucose oxidase/peroxidase (GOD/POD) method, which was compared with fasting blood sugar level. The individual effect of Glimepride + Metformin - Atovastatin + Fenofibrate on blood sugar level was tested after administration of single dose in animals, whereas the influence of repeated treatment of Atovastatin + Fenofibrate for seven days on the hypoglycemic effect of Glimepride + Metformin was studied.

\section{Statistical Data Analysis}

All values were expressed in mean $\pm \mathrm{SD}$. The data were analyzed by one way analysis of variance (ANOVA) using Graph Pad Prism Instat Software (version 6.00, Graph PadSoftware), using one way ANOVA followed by student $-t$ - test.

\section{RESULTS AND DISCUSSION}

On the basis of findings in the present study we found that the combination of test drugs has proved to be safe as far as the drug-drug interaction is concerned. The test and test both samples did not show any interaction. In behavioral study (Table 1) there were no extra ordinary changes observed. At the same time, effect of test samples on body weight (Table 2, Figure 1) of the exper-

\begin{tabular}{|c|c|c|c|c|c|}
\hline \multicolumn{7}{|c|}{ Table 1: Animal Behavioral activities. } \\
\hline Test & Sex & Control & Control $^{*}$ & Test & Test $^{*}$ \\
\hline \multirow{3}{*}{ Tremor } & M & + & - & + & - \\
\cline { 2 - 6 } & F & - & + & - & - \\
\hline \multirow{2}{*}{ Convulsion } & M & Normal & - & + & - \\
\cline { 2 - 7 } & F & - & - & - & - \\
\hline \multirow{2}{*}{ Salivation } & $\mathrm{M}$ & + & - & + & - \\
\cline { 2 - 7 } & $\mathrm{F}$ & - & - & - & + \\
\hline \multirow{2}{*}{ Diarrhoea } & $\mathrm{M}$ & + & - & + & - \\
\cline { 2 - 7 } & $\mathrm{F}$ & - & - & + & - \\
\hline \multirow{2}{*}{ Lethargy } & $\mathrm{M}$ & + & + & - & - \\
\cline { 2 - 7 } & $\mathrm{F}$ & + & - & - & - \\
\hline \multirow{2}{*}{ Sleep } & $\mathrm{M}$ & + & + & - & - \\
\cline { 2 - 7 } & $\mathrm{F}$ & + & - & - & - \\
\hline
\end{tabular}




\begin{tabular}{|c|c|c|c|c|c|}
\hline Time duration & Sex & Control & Control* $^{*}$ & Test & Test $^{*}$ \\
\hline \multirow[t]{2}{*}{0 Days } & M & $168.8 \pm 1.16$ & $172.4 \pm 1.99$ & $167.6 \pm 2.73$ & $166.4 \pm 1.36$ \\
\hline & $\mathrm{F}$ & $178.4 \pm 1.21$ & $173.2 \pm 1.46$ & $174.6 \pm 3.89$ & $177.2 \pm 2.29$ \\
\hline \multirow[t]{2}{*}{7 Days } & M & $170.2 \pm 1.02$ & $172.6 \pm 1.32$ & $165.3 \pm 2.1$ & $164.2 \pm 1.2$ \\
\hline & $\mathrm{F}$ & $181.2 \pm 1.23$ & $174.2 \pm 1.34$ & $170.5 \pm 1.5$ & $175.1 \pm 1.8$ \\
\hline \multirow[t]{2}{*}{14 Days } & M & $171.2 \pm 1.5$ & $173.6 \pm 1.5$ & $163.4 \pm 1.7$ & $161.2 \pm 2.43$ \\
\hline & $\mathrm{F}$ & $173.5 \pm 1.73$ & $176.2 \pm 1.34$ & $168.2 \pm 1.62$ & $172 \pm 2.13$ \\
\hline \multirow[t]{2}{*}{21 Days } & M & $173 \pm 2.02$ & $174.4 \pm 1.43$ & $160.2 \pm 1.03$ & $157.4 \pm 1.68$ \\
\hline & $\mathrm{F}$ & $174.5 \pm 1.32$ & $178.3 \pm 1.56$ & $166.3 \pm 1.21$ & $168.4 \pm 1.42$ \\
\hline \multirow[t]{2}{*}{28 Days } & M & $174.2 \pm 1.45$ & $175 \pm 1.7$ & $158 \pm 1.6$ & $155 \pm 1.89$ \\
\hline & $\mathrm{F}$ & $176.5 \pm 1.18$ & $180.2 \pm 1.2$ & $164.3 \pm 2.02$ & $164.3 \pm 2.03$ \\
\hline \multirow[t]{2}{*}{35 Days } & M & $176 \pm 1.8$ & $158.6 \pm 1.4$ & 160. \pm 0.22 & $176 \pm 1.8$ \\
\hline & $\mathrm{F}$ & $181 \pm 1.9$ & $166.4 \pm 1.78$ & $168.20 \pm 1.20$ & $181 \pm 1.9$ \\
\hline \multirow[t]{2}{*}{42 Days } & M & $175.2 \pm 1.32$ & $162.4 \pm 1.82$ & $172.02 \pm 1.30$ & $175.2 \pm 1.32$ \\
\hline & $\mathrm{F}$ & $182.3 \pm 1.53$ & $170 \pm 1.8$ & $178.40 \pm 1.42$ & $182.3 \pm 1.53$ \\
\hline
\end{tabular}

All the Values are expressed as mean \pm SEM. $(n=5)$

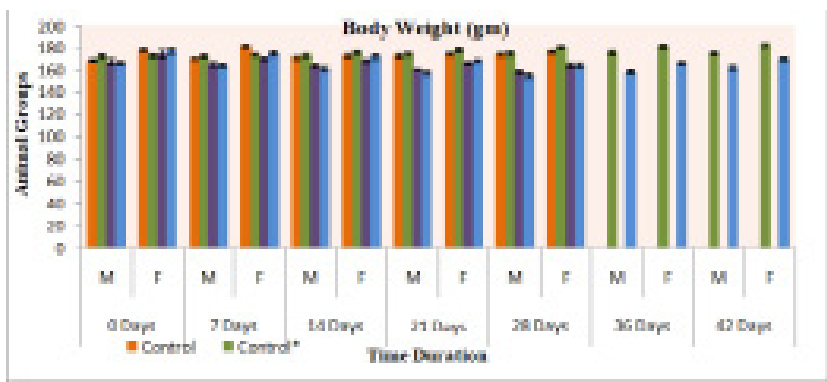

Figure 1: Animals mean body weight during dosing, Number of animals per group $=5$. All the Values are expressed as mean $\pm \operatorname{SEM}(n=5)$.

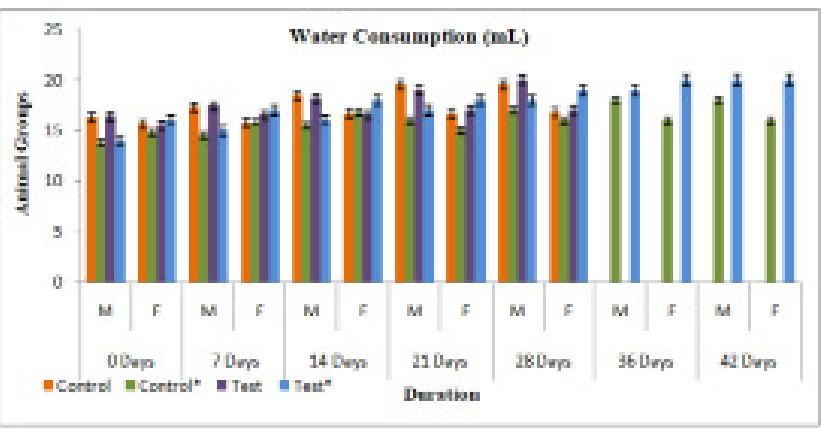

Figure 2: Animals mean Water consumption during dosing, Number of animals per group $=5$. All value are reported as Mean \pm SEM.

\begin{tabular}{|c|c|c|c|c|c|}
\hline Time duration & Sex & Control & Control* & Test & Test* \\
\hline \multirow[t]{2}{*}{0 Days } & M & $16.3 \pm 1.64$ & $13.8 \pm 1.03$ & $16.4 \pm 1.07$ & $14 \pm 0.7$ \\
\hline & $\mathrm{F}$ & $15.6 \pm 0.3$ & $14.8 \pm 1.4$ & $15.5 \pm 1.75$ & $16 \pm 0.3$ \\
\hline \multirow[t]{2}{*}{7 Days } & M & $17.3 \pm 1.75$ & $14.5 \pm 0.25$ & $17.4 \pm 0.07$ & $15 \pm 0.5$ \\
\hline & $\mathrm{F}$ & $15.8 \pm 1.3$ & $15.8 \pm 1.07$ & $16.5 \pm 0.75$ & $17 \pm 1.2$ \\
\hline \multirow[t]{2}{*}{14 Days } & $M$ & $18.5 \pm 1.75$ & $15.5 \pm 0.75$ & $18.2 \pm 1.65$ & $16 \pm 0.5$ \\
\hline & $F$ & $16.7 \pm 0.05$ & $16.8 \pm 1.7$ & $16.5 \pm 1.5$ & $18 \pm 0.1$ \\
\hline \multirow[t]{2}{*}{21 Days } & M & $19.5 \pm 0.25$ & $16 \pm 1.5$ & $19.0 \pm 0.5$ & $17 \pm 0.2$ \\
\hline & $\mathrm{F}$ & $16.7 \pm 0.5$ & $15 \pm 1.2$ & $17 \pm 0.57$ & $18 \pm 1.5$ \\
\hline \multirow[t]{2}{*}{28 Days } & $M$ & $19.5 \pm 1.78$ & $17 \pm 0.25$ & $20 \pm 1.05$ & $18 \pm 1.5$ \\
\hline & $\mathrm{F}$ & $16.8 \pm 1.4$ & $16 \pm 0.5$ & $17 \pm 0.25$ & $19 \pm 1.35$ \\
\hline \multirow[t]{2}{*}{35 Days } & M & $17.2 \pm 0.24$ & $18 \pm 0.5$ & $18 \pm 0.24$ & $19 \pm 0.5$ \\
\hline & $\mathrm{F}$ & $18.2 \pm 0.62$ & $16 \pm 1.75$ & $16 \pm 1.2$ & $20 \pm 1.5$ \\
\hline \multirow[t]{2}{*}{42 Days } & $M$ & $16.5 \pm 0.40$ & $18 \pm 2.5$ & $16.5 \pm 0.62$ & $20 \pm 1.0$ \\
\hline & $\mathrm{F}$ & $19.4 \pm 0.62$ & $16 \pm 0.75$ & $18.2 \pm 0.20$ & $20 \pm 1.4$ \\
\hline
\end{tabular}

All the Values are expressed as mean \pm SEM. $(n=5)$ 


\begin{tabular}{|c|c|c|c|c|c|}
\hline Time duration & Sex & Control & Control* & Test & Test $^{*}$ \\
\hline \multirow[t]{2}{*}{0 Days } & $M$ & $16 \pm 1.58$ & $17 \pm 2.1$ & $16 \pm 1.60$ & $17.5 \pm 1.4$ \\
\hline & $\mathrm{F}$ & $16.2 \pm 1.69$ & $18 \pm 1.71$ & $16.5 \pm 1.70$ & $18.2 \pm 1.55$ \\
\hline \multirow[t]{2}{*}{7 Days } & $M$ & $17 \pm 2.1$ & $18 \pm 1.60$ & $17.5 \pm 1.49$ & $18.2 \pm 4.5$ \\
\hline & $F$ & $17 \pm 1.71$ & $16.5 \pm 1.70$ & $17.8 \pm 1.55$ & $19.2 \pm 2.3$ \\
\hline \multirow[t]{2}{*}{14 Days } & M & $18 \pm 2.5$ & $19 \pm 1.69$ & $18.1 \pm 2.6$ & $19.2 \pm 1.56$ \\
\hline & $F$ & $17 \pm 2.83$ & $17.2 \pm 1.70$ & $16 \pm 1.23$ & $20 \pm 1.4$ \\
\hline \multirow[t]{2}{*}{21 Days } & $M$ & $19 \pm 4.5$ & $20 \pm 2.5$ & $16 \pm 1.8$ & $20 \pm 2.75$ \\
\hline & $F$ & $18 \pm 2.5$ & $16 \pm 2.2$ & $17 \pm 2.5$ & $21 \pm 2.3$ \\
\hline \multirow[t]{2}{*}{28 Days } & $\mathrm{M}$ & $20 \pm 3.6$ & $19 \pm 4.5$ & $19 \pm 4.5$ & $21 \pm 8.95$ \\
\hline & $\mathrm{F}$ & $19 \pm 3.5$ & $17 \pm 4.7$ & $16 \pm 3.75$ & $22 \pm 0.5$ \\
\hline \multirow[t]{2}{*}{35 Days } & $\mathrm{M}$ & $16 \pm 2.4$ & $20.2 \pm 4.3$ & $17 \pm 2.4$ & $22 \pm 6.5$ \\
\hline & $\mathrm{F}$ & $18 \pm 3.2$ & $18 \pm 2.7$ & $16 \pm 3.4$ & $23 \pm 1.75$ \\
\hline \multirow[t]{2}{*}{42 Days } & M & $17 \pm 4.2$ & $21 \pm 2.5$ & $18 \pm 5.4$ & $23 \pm 7.5$ \\
\hline & $\mathrm{F}$ & $16 \pm 2.6$ & $19 \pm 5.5$ & $17 \pm 2.4$ & $23 \pm 2.75$ \\
\hline
\end{tabular}

All the Values are expressed as mean \pm SEM. $(n=5)$

\begin{tabular}{|c|c|c|c|c|c|}
\hline Time Duration & Sex & Control & Control* & Test & Test* \\
\hline \multirow[t]{2}{*}{0 Days } & M & $13.32 \pm 0.14$ & $13.58 \pm 0.15$ & $13.76 \pm 0.13$ & $13.92 \pm 0.11$ \\
\hline & $\mathrm{F}$ & $12.46 \pm 0.14$ & $12.58 \pm 0.15$ & $12.46 \pm 0.21$ & $12.76 \pm 0.15$ \\
\hline \multirow[t]{2}{*}{7 Days } & $M$ & $13.20 \pm 0.13$ & $13.62 \pm 0.17$ & $13.52 \pm 0.17$ & $14.30 \pm 0.20$ \\
\hline & $\mathrm{F}$ & $12.80 \pm 0.12$ & $12.70 \pm 0.12$ & $12.52 \pm 0.18$ & $13.20 \pm 0.15$ \\
\hline \multirow[t]{2}{*}{14 Days } & M & $13.02 \pm 0.23$ & $13.40 \pm 0.17$ & $13.61 \pm 0.22$ & $13.90 \pm 0.16$ \\
\hline & $\mathrm{F}$ & $12.50 \pm 0.18$ & $12.60 \pm 0.15$ & $12.27 \pm 0.09$ & $12.90 \pm 0.21$ \\
\hline \multirow[t]{2}{*}{21 Days } & M & $13.30 \pm 0.23$ & $13.20 \pm 0.16$ & $13.90 \pm 0.11$ & $14.30 \pm 0.13$ \\
\hline & $\mathrm{F}$ & $12.6 \pm 0.13$ & $12.60 \pm 0.19$ & $12.80 \pm 0.13$ & $13.20 \pm 0.11$ \\
\hline \multirow[t]{2}{*}{28 Days } & M & $13.70 \pm 0.12$ & $13.80 \pm 0.12$ & $13.60 \pm 0.15$ & $13.67 \pm 0.19$ \\
\hline & $\mathrm{F}$ & $12.30 \pm 0.15$ & $12.80 \pm 0.13$ & $12.50 \pm 0.14$ & $12.93 \pm 0.18$ \\
\hline
\end{tabular}

All the Values are expressed as mean \pm SEM. $(n=5)$

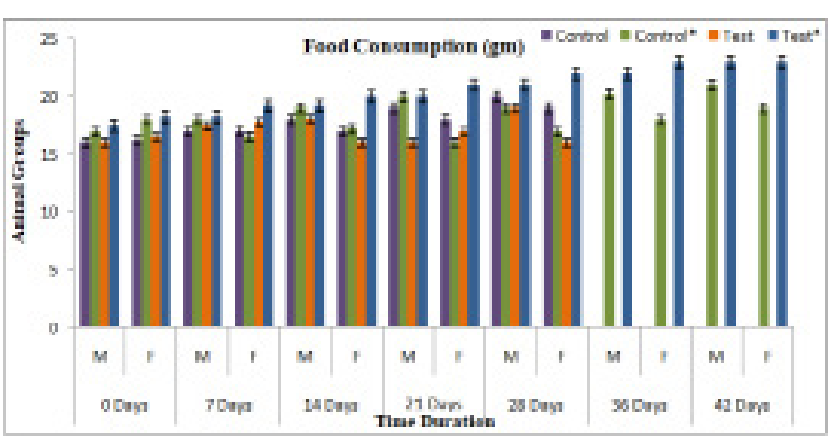

Figure 3: Animals mean food consumption during dosing, Number of animals per group $=5$. All the Values are expressed as mean $\pm \operatorname{SEM}(n=5)$. imental animals showed some change. At the beginning of the experiment the observed body weight, for control was $168.8 \pm 1.16$, but at the end of 42 days, increase in body weight was observed. Similarly, water consumption (Table 3 Figure 2) and food consumption (Table 4, Figure 3) was found to be increased very less after 35 days. Consequently, Hematological parameters were also observed with no marked increase in the hemoglobin level (Table 5, Figure 4) during the activity. A slight increase in neutrophils level was observed (Table 6, Figure 5), Changes in the platelet level (Table 7, Figure 6) at the end of the activity was also not much signifi- 


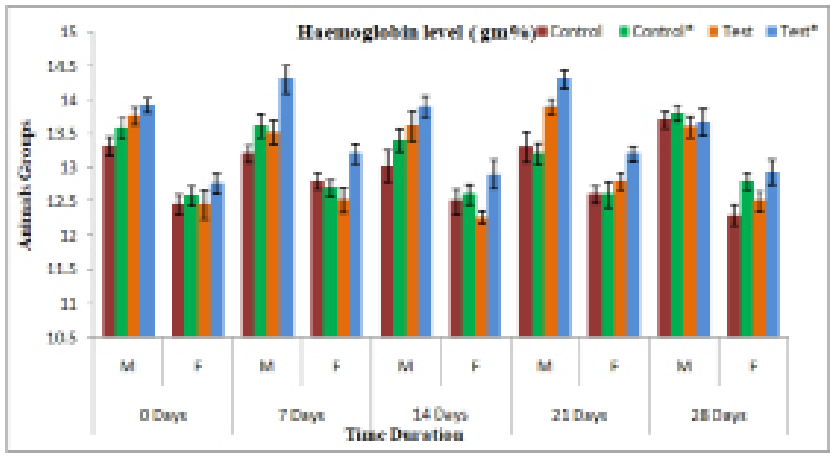

Figure 4: Animals mean Haemoglobin level during dosing, number of animals per group $=5$. All the Values are expressed as mean $\pm \operatorname{SEM}(n=5)$.

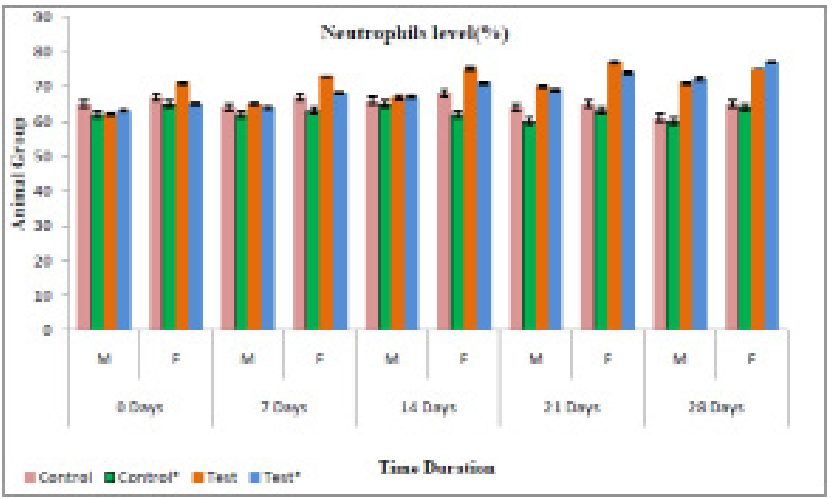

Figure 5: Animals mean neutrophils level during dosing, Number of animals per group $=5$. All the Values are expressed as mean $\pm \operatorname{SEM}(n=5)$.

\begin{tabular}{|c|c|c|c|c|c|}
\hline Time Duration & Sex & Control & Control* $^{*}$ & Test & Test $^{*}$ \\
\hline \multirow[t]{2}{*}{0 Days } & M & $65 \pm 0.411$ & $62 \pm 0.412$ & $62 \pm 0.313$ & $63 \pm 0.412$ \\
\hline & $\mathrm{F}$ & $67 \pm 0.314$ & $65 \pm 0.513$ & $71 \pm 0.314$ & $65 \pm 0.310$ \\
\hline \multirow[t]{2}{*}{7 Days } & M & $64 \pm 0.412$ & $62 \pm 0.515$ & $65 \pm 0.211$ & $64 \pm 0.511$ \\
\hline & $\mathrm{F}$ & $67 \pm 0.514$ & $63 \pm 0.514$ & $73 \pm 0.112$ & $68 \pm 0.410$ \\
\hline \multirow[t]{2}{*}{14 Days } & M & $66 \pm 0.311$ & $65 \pm 0.312$ & $67 \pm 0.513$ & $67 \pm 0.317$ \\
\hline & $\mathrm{F}$ & $68 \pm 0.412$ & $62 \pm 0.410$ & $75 \pm 0.513$ & $71 \pm 0.409$ \\
\hline \multirow[t]{2}{*}{21 Days } & $\mathrm{M}$ & $64 \pm 0.418$ & $60 \pm 0.513$ & $70 \pm 0.412$ & $69 \pm 0.412$ \\
\hline & $\mathrm{F}$ & $65 \pm 0.313$ & $63 \pm 0.415$ & $77 \pm 0.315$ & $74 \pm 0.317$ \\
\hline \multirow[t]{2}{*}{28 Days } & M & $61 \pm 0.413$ & $60 \pm 0.518$ & $71 \pm 0.312$ & $72 \pm 0.514$ \\
\hline & $\mathrm{F}$ & $65 \pm 0.517$ & $64 \pm 0.313$ & $75 \pm 0.016$ & $77 \pm 0.212$ \\
\hline
\end{tabular}

All the Values are expressed as mean \pm SEM. $(n=5)$

\begin{tabular}{|c|c|c|c|c|c|}
\hline Time Duration & Sex & Control & Control $^{*}$ & Test & Test $^{*}$ \\
\hline \multirow[t]{2}{*}{0 Days } & M & $3.75 \pm 0.014$ & $3.70 \pm 0.008$ & $3.75 \pm 0.005$ & $3.60 \pm 0.003$ \\
\hline & $\mathrm{F}$ & $3.80 \pm 0.006$ & $3.90 \pm 0.005$ & $3.90 \pm 0.016$ & $3.80 \pm 0.006$ \\
\hline \multirow[t]{2}{*}{7 Days } & $M$ & $3.79 \pm 0.015$ & $3.75 \pm 0.004$ & $3.85 \pm 0.004$ & $3.85 \pm 0.004$ \\
\hline & $\mathrm{F}$ & $3.85 \pm 0.006$ & $3.80 \pm 0.006$ & $4.20 \pm 0.003$ & $4.10 \pm 0.016$ \\
\hline \multirow[t]{2}{*}{14 Days } & $M$ & $3.80 \pm 0.017$ & $3.60 \pm 0.007$ & $4.10 \pm 0.004$ & $4.15 \pm 0.005$ \\
\hline & $\mathrm{F}$ & $3.80 \pm 0.006$ & $3.90 \pm 0.006$ & $4.30 \pm 0.025$ & $4.30 \pm 0.006$ \\
\hline \multirow[t]{2}{*}{21 Days } & M & $3.65 \pm 0.040$ & $3.50 \pm 0.014$ & $4.25 \pm 0.004$ & $4.25 \pm 0.004$ \\
\hline & $\mathrm{F}$ & $3.70 \pm 0.006$ & $3.70 \pm 0.005$ & $4.45 \pm 0.005$ & $4.45 \pm 0.005$ \\
\hline \multirow[t]{2}{*}{28 Days } & M & $3.90 \pm 0.004$ & $3.70 \pm 0.004$ & $4.30 \pm 0.009$ & $4.30 \pm 0.005$ \\
\hline & $\mathrm{F}$ & $3.50 \pm 0.005$ & $3.80 \pm 0.012$ & $4.50 \pm 0.006$ & $4.50 \pm 0.006$ \\
\hline
\end{tabular}

All the Values are expressed as mean \pm SEM. $(n=5)$ 


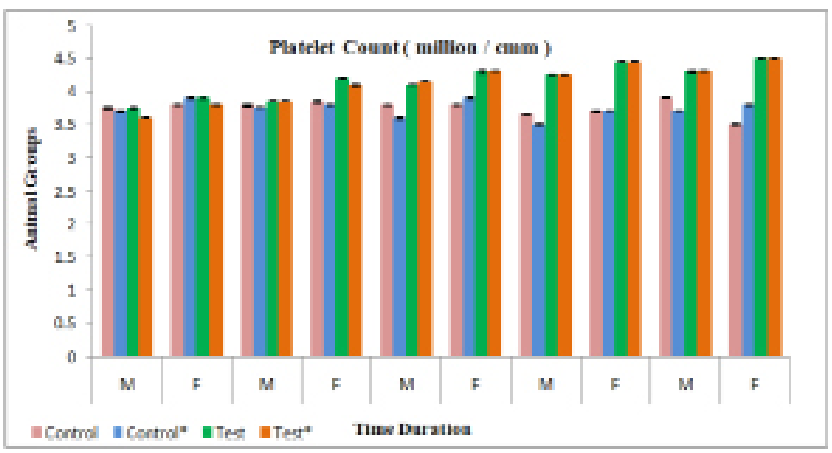

Figure 6: Animals mean platelets level during dosing, Number of animals per group $=5$. All the Values are expressed as mean $\pm \operatorname{SEM}(n=5)$.

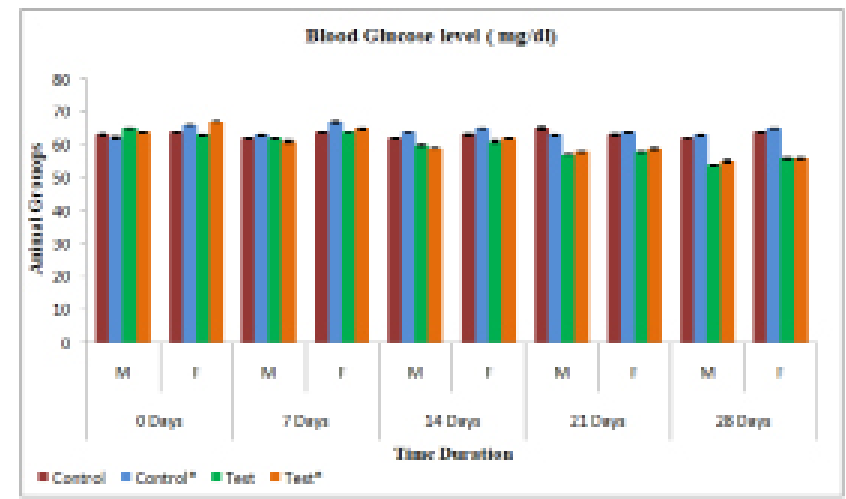

Figure 7: Animals mean glucose level during dosing, number of animals per group $=5$. All the Values are expressed as mean $\pm \operatorname{SEM}(n=5)$.

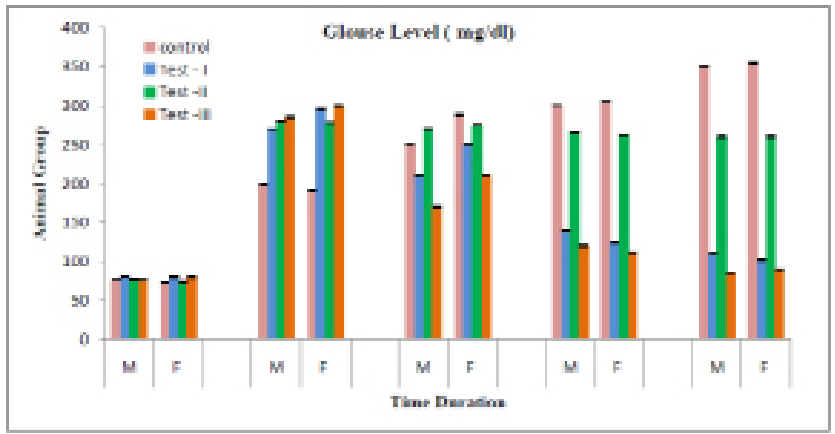

Figure 8: Animals mean glucose level during dosing, Number of animals per group $=5$

All the Values are expressed as mean \pm SEM $(n=5)$.

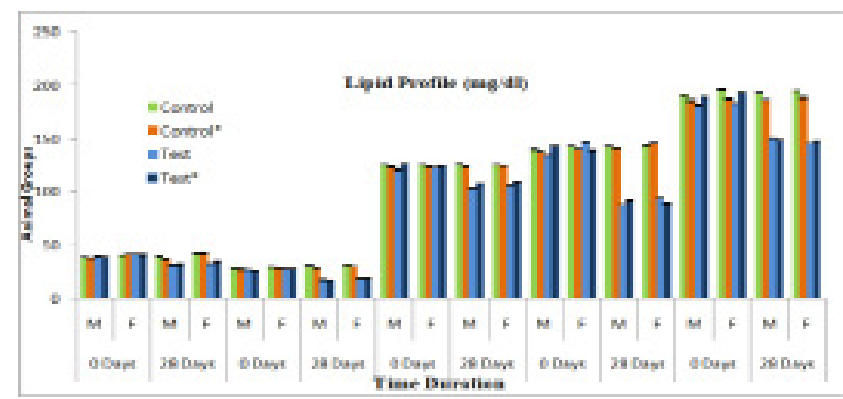

Figure 9: Animals Mean lipid profile during dosing, Number of animals per group $=5$. All the Values are expressed as mean $\pm \operatorname{SEM}(n=5)$.

\begin{tabular}{|c|c|c|c|c|c|}
\hline Time Duration & Sex & Control & Control $^{*}$ & Test & Test ${ }^{*}$ \\
\hline \multirow{2}{*}{0 Day } & M & $76 \pm 0.054$ & $81 \pm 0.23$ & $77 \pm 0.67$ & $76 \pm 0.12$ \\
\hline & $\mathrm{F}$ & $73 \pm 0.23$ & $80 \pm 0.56$ & $74 \pm 0.34$ & $80 \pm 0.34$ \\
\hline \multirow{2}{*}{$24 \mathrm{Hr}$} & M & $198 \pm 0.45$ & $270 \pm 0.12$ & $280 \pm 0.23$ & $285 \pm 0.234$ \\
\hline & $\mathrm{F}$ & $191 \pm 0.67$ & $295 \pm 0.34$ & $278 \pm 0.34$ & $300 \pm 0.56$ \\
\hline \multirow{2}{*}{$7^{\text {th }}$ Day } & M & $250 \pm 0.23$ & $210 \pm 0.198$ & $270 \pm 0.987$ & $170 \pm 0.78$ \\
\hline & $\mathrm{F}$ & $288 \pm 0.11$ & $250 \pm 0.14$ & $275 \pm 0.34$ & $210 \pm 0.12$ \\
\hline \multirow{2}{*}{21 Day } & M & $300 \pm 0.234$ & $140 \pm 0.13$ & $265 \pm 0.56$ & $120 \pm 0.34$ \\
\hline & $\mathrm{F}$ & $305 \pm 0.123$ & $124 \pm 0.167$ & $262 \pm 0.23$ & $112 \pm 0.124$ \\
\hline \multirow{2}{*}{28 Day } & M & $350 \pm 0.87$ & $110 \pm 0.34$ & $260 \pm 0.45$ & $85 \pm 0.16$ \\
\hline & $\mathrm{F}$ & $355 \pm 0.178$ & $102 \pm 0.234$ & $260 \pm 0.23$ & $90 \pm 0.012$ \\
\hline
\end{tabular}

All the Values are expressed as mean \pm SEM. $(n=5)$ 


\begin{tabular}{|c|c|c|c|c|c|c|}
\hline Parameter & Time Duration & Sex & Control & Control* & Test & Test $^{*}$ \\
\hline \multirow{4}{*}{ HDL } & \multirow[t]{2}{*}{0 Days } & M & $38 \pm 0.25$ & $37.2 \pm 0.27$ & $39.5 \pm 0.33$ & $38.2 \pm 0.34$ \\
\hline & & $F$ & $40.2 \pm 0.34$ & $41.3 \pm 0.31$ & $41.3 \pm 0.2$ & $40.6 \pm 0.32$ \\
\hline & \multirow[t]{2}{*}{28 Days } & M & $39.2 \pm 0.23$ & $36.4 \pm 0.27$ & $30.7 \pm 0.33$ & $31.6 \pm 0.34$ \\
\hline & & $F$ & $42.1 \pm 0.34$ & $42.4 \pm 0.31$ & $32.3 \pm 0.2$ & $34.3 \pm 0.32$ \\
\hline \multirow{4}{*}{ VLDL } & \multirow[t]{2}{*}{0 Days } & M & $27.82 \pm 0.23$ & $26.35 \pm 0.27$ & $26 \pm 0.33$ & $25 \pm 0.34$ \\
\hline & & $F$ & $28.5 \pm 0.34$ & $27.5 \pm 0.31$ & $26.5 \pm 0.2$ & $27 \pm 0.32$ \\
\hline & \multirow[t]{2}{*}{28 Days } & M & $30 \pm 0.28$ & $28 \pm 0.27$ & $17.48 \pm 0.33$ & $17 \pm 0.34$ \\
\hline & & $\mathrm{F}$ & $31.25 \pm 0.34$ & $29.2 \pm 0.31$ & $18.35 \pm 0.2$ & $18.25 \pm 0.32$ \\
\hline \multirow{4}{*}{ LDL } & \multirow[t]{2}{*}{0 Days } & M & $124.18 \pm 0.27$ & $122.35 \pm 0.27$ & $120.35 \pm 0.33$ & $125.2 \pm 0.34$ \\
\hline & & $\mathrm{F}$ & $124.5 \pm 0.34$ & $122.36 \pm 0.3$ & $122.05 \pm 0.2$ & $123.5 \pm 0.36$ \\
\hline & \multirow[t]{2}{*}{28 Days } & M & $125.3 \pm 0.23$ & $122.7 \pm 0.27$ & $102.45 \pm 0.33$ & $106.5 \pm 0.34$ \\
\hline & & $\mathrm{F}$ & $124.45 \pm 0.34$ & $123.8 \pm 0.31$ & $104.9 \pm 0.2$ & $109.1 \pm 0.2$ \\
\hline \multirow{4}{*}{ S-TG } & \multirow[t]{2}{*}{0 Days } & M & $139.1 \pm 0.26$ & $136.7 \pm 0.28$ & $133.5 \pm 0.33$ & $142.3 \pm 0.34$ \\
\hline & & $\mathrm{F}$ & $142.3 \pm 0.36$ & $139.5 \pm 0.35$ & $146.4 \pm 0.2$ & $138.4 \pm 0.34$ \\
\hline & \multirow[t]{2}{*}{28 Days } & M & $142.5 \pm 0.23$ & $140.5 \pm 0.27$ & $87.4 \pm 0.33$ & $91.2 \pm 0.34$ \\
\hline & & $\mathrm{F}$ & $143.4 \pm 0.34$ & $145.5 \pm 0.31$ & $93.4 \pm 0.2$ & $88.5 \pm 0.34$ \\
\hline \multirow{4}{*}{$\mathrm{S}-\mathrm{CHOL}$} & \multirow[t]{2}{*}{0 Days } & M & $190 \pm 0.23$ & $185.4 \pm 0.27$ & $180.7 \pm 0.33$ & $188.4 \pm 0.34$ \\
\hline & & $\mathrm{F}$ & $195.2 \pm 0.34$ & $187.2 \pm 0.33$ & $182.25 \pm 0.2$ & $192 \pm 0.32$ \\
\hline & \multirow[t]{2}{*}{28 Days } & M & $192 \pm 0.23$ & $186 \pm 0.27$ & $150 \pm 0.33$ & $148.5 \pm 0.34$ \\
\hline & & $\mathrm{F}$ & $194 \pm 0.34$ & $188 \pm 0.3$ & $145 \pm 0.2$ & $147.5 \pm 0.32$ \\
\hline
\end{tabular}

All the Values are expressed as mean \pm SEM. $(n=5)$.

\begin{tabular}{|c|c|c|c|c|c|}
\hline Time Duration & Sex & Control & Control* & Test & Test $^{*}$ \\
\hline \multirow[t]{2}{*}{0 Days } & M & $25 \pm 0.311$ & $24 \pm 0.332$ & $25 \pm 0.313$ & $26 \pm 0.532$ \\
\hline & $\mathrm{F}$ & $26 \pm 0.314$ & $27 \pm 0.452$ & $27 \pm 0.314$ & $28 \pm 0.367$ \\
\hline \multirow[t]{2}{*}{7 Days } & M & $26 \pm 0.422$ & $24 \pm 0.515$ & $22 \pm 0.3167$ & $24 \pm 0.522$ \\
\hline & $\mathrm{F}$ & $26 \pm 0.512$ & $25 \pm 0.534$ & $25 \pm 0.112$ & $25 \pm 0.418$ \\
\hline \multirow[t]{2}{*}{14 Days } & M & $27 \pm 0.331$ & $26 \pm 0.312$ & $20 \pm 0.513$ & $21 \pm 0.317$ \\
\hline & $\mathrm{F}$ & $28 \pm 0.412$ & $27 \pm 0.41$ & $24 \pm 0.553$ & $24 \pm 0.439$ \\
\hline \multirow[t]{2}{*}{21 Days } & M & $25 \pm 0.418$ & $26 \pm 0.513$ & $19 \pm 0.412$ & $19 \pm 0.412$ \\
\hline & $\mathrm{F}$ & $27 \pm 0.363$ & $26 \pm 0.425$ & $23 \pm 0.315$ & $22 \pm 0.317$ \\
\hline \multirow[t]{2}{*}{28 Days } & M & $26 \pm 0.413$ & $25 \pm 0.518$ & $17 \pm 0.312$ & $19 \pm 0.534$ \\
\hline & $\mathrm{F}$ & $26 \pm 0.513$ & $28 \pm 0.313$ & $20 \pm 0.016$ & $21 \pm 0.442$ \\
\hline
\end{tabular}




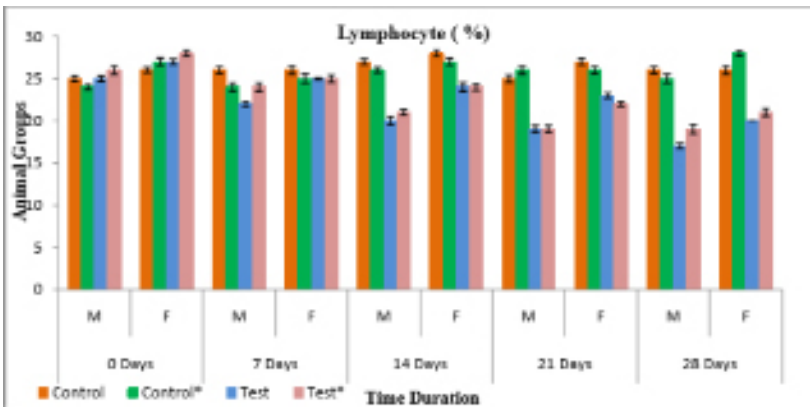

Figure 10: Animals Lymphocyte \% during dosing, Number of animals per group $=5$. All the Values are expressed as mean \pm SEM $(n=5)$.

(A)
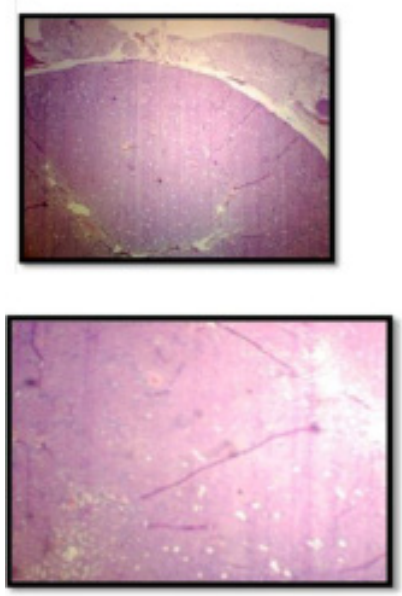

(C)

Figure 11: Histopathological observation of kidney tissue in rats (magnification 100x).

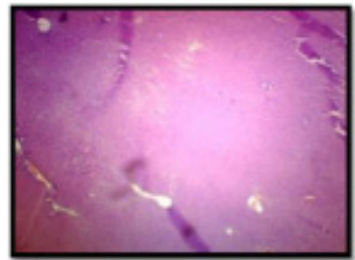

(A)

(C)

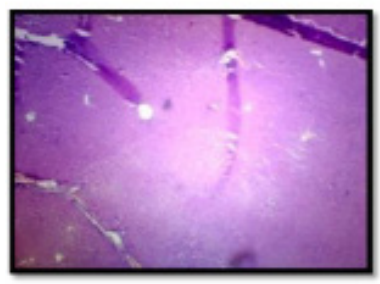

Figure 12: Histopathological observation of liver tissue in rats (magnification 100x). (a)

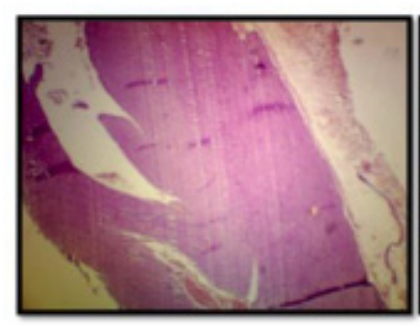

(b)
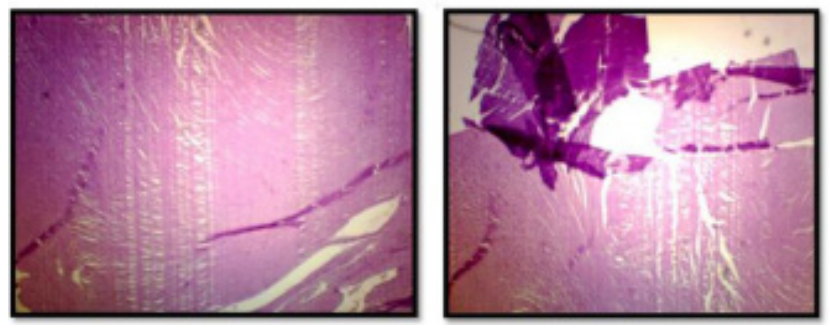

Figure 13: Histopathological observation of heart tissue in rats (magnification 100x).

Note: In the Figure (A) Histopathology of section of control group (B) Histopathology of section of control* group (C) Histopathology of section of Test group(D) Histopathology of section of Test ${ }^{*}$ group

cant. The result of most important parameter, blood glucose level (Table 8, Figure 7,8) showed a significant decrease. In lipid profile (Table 9, Figure 9), HDL level, VLDL level, LDL level, S-TG and S-Cholesterol level were decreased in a very significant manner. A slight decrease in lymphocyte level (Table 10, Figure 10) was observed i.e. showed not much significant increase.

Histopathological observation of kidney liver and heart tissues (Figures 11-13) suggests that the combination of these Antidiabetic and hypolipidemic drugs is safe in all aspects for the diabetic patients.

\section{ACKNOWLEDGEMENT}

The authors are thankful to all the members of the management of Columbia Institute of Pharmacy, Raipur, for providing all facilities to conduct this experimental work.

\section{CONFLICT OF INTEREST}

The authors declare no conflict of interest

\section{Ethical permission for pre-clinical study}

The protocol used in the study was permitted by Institutional animal ethics committee (IAEC) approved by CPCSEANew Delhi, Columbia Institute of Pharmacy, Raipur (C.G.) approval No is CIP/IAEC/2017/085 and Regd.No.1321/PO/ReBi/S/10/CPCSEA. 


\section{ABBREVIATIONS}

IAEC: Institutional Animal Ethicalcommittee; CIP: Columbia Institute of Pharmacy; CPCSEA: Committee for the Purpose of Control and Supervision of Experiments on Animals; STZ: Streptozotocin; GOD: Glucose oxidase; POD: Glucose peroxidase; SD: Standard deviation.

\section{REFERENCES}

1. Sharma HL, Sharma KK. Principles of Pharmacology. $1^{\text {st }}$ edition. Paras medical publisher; 2007;125-50.

2. Shukla SS, Sharma A, Tiwari RK, Sharma V, Pandey RK. Antidiabetic Activity of an Ayurvedic Formulation Chaturmukha Rasa: A Mechanism Based Study. Journal of Pharmacopunture. 2019;22(2):115-21.

3. Tripathi KD. Essentials of medical pharmacology. JP Medical Ltd. India. 2013.

4. Satoskar RS, Bhandarkar SD, Rege NN. Pharmacology and pharmacotherapeutics. 13 $3^{\text {th }}$ edition. Popular Prakashan; 2013.

5. Sultanpur CM, Reddy NS, Kumar S, Satyanarayana S, Kumar KE. Drug-drug interaction between pravastatin and gemfibrozil (antihyperlipidemic) with gliclazide (antidiabetic) in rats. J Young Pharm. 2010;2(2):152-5.

6. Parameshappa B, Rao NV, Gouda TS, Sen S, Chakraborty R, Basha MA, et al. A study on drug-drug interaction between anti-hypertensive drug (propranolol) and anti-diabetic drug (glipizide). Ann Biol Res. 2010;1(3):3540.

7. Dave V, Sharma R, Sharma S, Jain P, Yadav S. Experimental Models on Diabetes: A Comprehensive Review. International Journal of Advances in Pharmaceutical Sciences. 2013;4(1):1-8.

8. Dewangan H, Tiwari RK, Sharma V, Shukla SS, Satapathy T, Pandey R. Past and Future of in-vitro and in-vivo Animal Models for Diabetes: A Review. Indian Journal of Pharmaceutical Education and Research. 2017;51(4S):s522-30.

9. Sreemantula S, Kilari EK, Vardhan VA, Jaladi R. Influence of antioxidant (L-ascorbic acid) on tolbutamide induced hypoglycaemia/antihyperglycaemia in normal and diabetic rats. BMC Endocrine Disorders. 2005;5(1):1-4.

10. Kuhad A, Sethi R, Chopra K. Lycopene attenuates diabetes-associated cognitive decline in rats. Life Sciences. 2008;83(3):128-34.
11. Piero MN, Nzaro GM, Njagi JM. Diabetes mellitus-a devastating metabolic disorder. Asian journal of biomedical and pharmaceutical sciences. 2015;5(40):1.

12. Baynes HW. Classification, Pathophysiology, Diagnosis and Management of Diabetes Mellitus. J Diabetes Metab. 2015;6(541):1-9.

13. Homsi AMF, Lukic ML. An Update on the pathogenesis of Diabetes Mellitus. UAE University, Al Ain, United Arab Emirates; 1992.

14. Diabetes Control and Complications Trial. Epidemiology of Diabetes Interventions and Complications Research Group. Intensive diabetes therapy and carotid intima-media thickness in type 1 diabetes mellitus. N Engl J Med. 2003;348:2294-303.

15. Ozougwu JC, Obimba KC, Belonwu CD, Unakalamba CB. The pathogenesis and pathophysiology of type 1 and type 2 diabetes mellitus. J Physiol Pathophysiol. 2013;4(4):46-57.

16. American Diabetes Association. Diagnosis and classification of diabetes mellitus. Diabetes Care. 2014;37:S81-90.

17. Pietri A, Dunn FL, Raskin P. The effect of improved diabetic control on plasma lipid and lipoprotein levels: A comparison of conventional therapy and continuous subcutaneous insulin infusion. Diabetes. 1980;29(12):1001-5.

18. Dunn FL. Hyperlipidemia and diabetes. Medical Clinics of North America. 1982;66(6):1347-60.

19. Garcia MJ, McNamara PM, Gordon T, Kannell WB. Morbidity and mortality in diabetics in the Framingham population: sixteen year follow-up study. Diabetes. 1974;23(2):105-11.

20. Pandey RK, Sharma V, Tiwari RK, Shukla SS. Diabetic Foot Ulcer; Management and Novel Treatment Approaches. Indo American Journal of Pharmaceutical Sciences. 2018;5(4):2824-8.

21. Kannel WB, McGee DL. Diabetes and cardiovascular risk factors: The Framingham study. Circulation. 1979;59(1):8-13.

22. Ginsberg HN. Very low density lipoprotein metabolism in diabetes mellitus. Diabetes/Metabolism Research and Reviews. 1987;3(2):571-89.

23. Sosenko JM, Breslow JL, Miettinen OS, Gabbay KH. Hyperglycemia and plasma lipid levels: a prospective study of young insulin-dependent diabetic patients. New England Journal of Medicine. 1980;302(12):650-4.

24. Sosenko JM, Breslow J, Miettinen OS, Gabbay KH. Hyperglycemia and plasma lipid levels: covariations in insulin-dependent diabetes. Diabetes Care. 1982;5(1):40-3.

25. Pietri A, Dunn FL, Raskin P. The effect of improved diabetic control on plasma lipid and lipoprotein levels: A comparison of conventional therapy and continuous subcutaneous insulin infusion. Diabetes. 1980;29(12):1001-5.

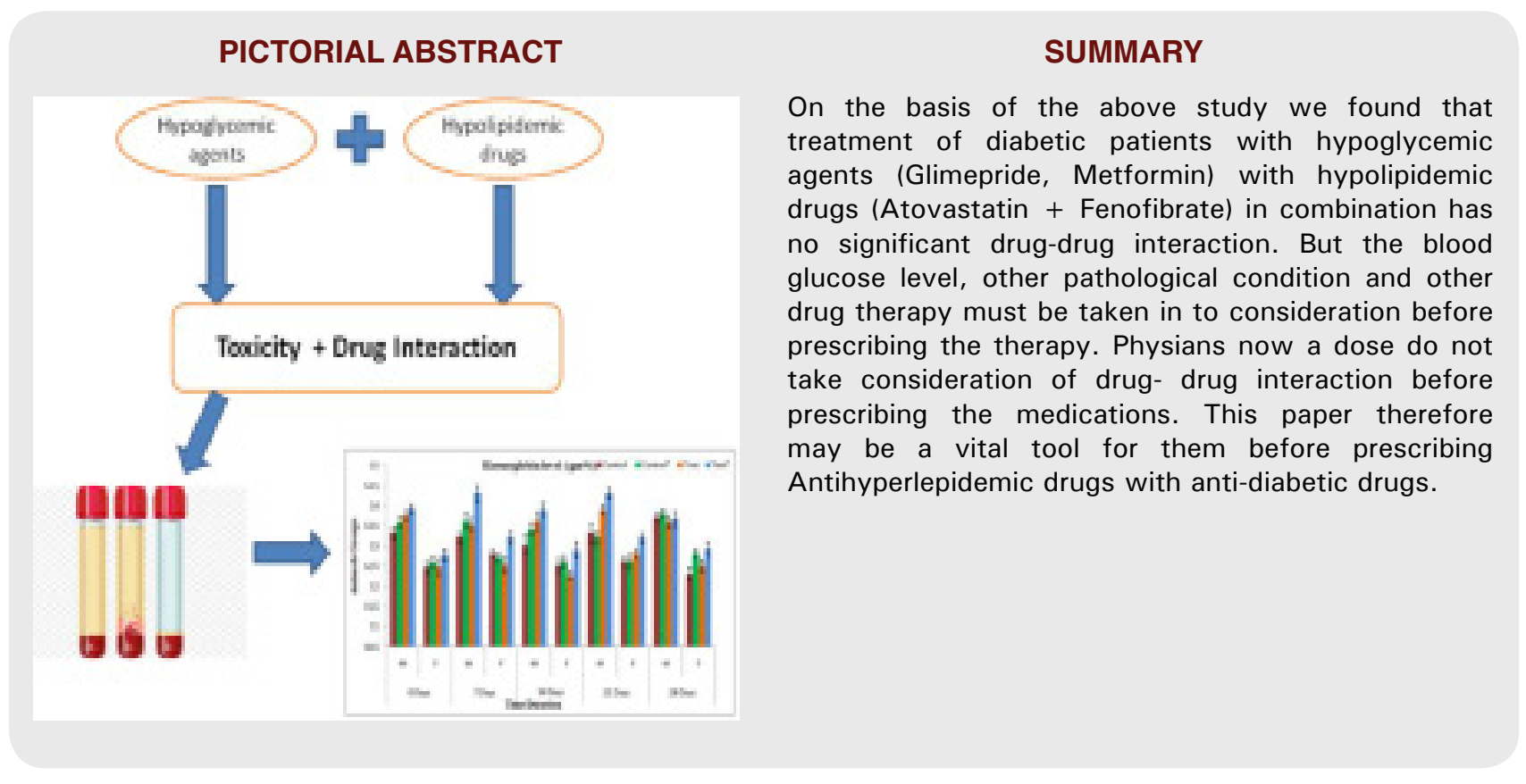




\section{About Authors}

Mrs. Hemlata Dewangan is working as Post Graduate Scholar in Department of Pharmacology, Columbia Institute of Pharmacy Raipur, (C.G). He is working on treatment of Diabetes.

Mr. Raj Kumar Tiwari is working as Ph.D research scholar in Department of Pharmacology, Columbia Institute of Pharmacy Raipur, (C.G). He is working on treatment of Diabetes.

Mr.Vikash Sharma is working as PhD research scholar in Department of Pharmacology, Columbia Institute of Pharmacy Raipur, (C.G). He is working on autoimmune disorders of poly-herbal formulation.

Prof. Shiv Shankar Shukla is working as Professor, Department of Pharmaceutical Analysis and Quality Assurance at Columbia Institute of Pharmacy. Author's area of interest includes instrumentation, standardization, validation and analysis of herbal and synthetic compounds.

Prof. Ravindra Kumar Pandey is working as Professor, Department of Pharmacognosy at Columbia Institute of Pharmacy, Raipur, (C.G). Author's area of interest includes conventional medicine system, standardization and chemical fingerprinting method development of Ayurvedic formulation and herbal formulation.

Cite this article: Dewangan H, Tiwari RK, Sharma V, Shukla SS, Pandey RK. Assessment of Toxicity and Drug-Drug Interaction of Combination Therapy Prescribed by Physicians/ Clinicians for Treatment of Diabetes using Experimental Animals. Indian J of Pharmaceutical Education and Research. 2021;55(2s):s605-s615. 